\title{
Ecballocystis pulvinata Bohlin (Hormotilaceae, Chlorophyta): morfologia externa e ocorrência em ambientes lóticos da região Sudeste do Brasil
}

\author{
Jeferson Luizi Pereira ${ }^{1,2}$, Luis Henrique Zanini Branco ${ }^{1}$ e Orlando Necchi Junior ${ }^{1}$
}

Recebido: 31.01.2011; aceito: 10.11.2011

\begin{abstract}
Ecballocystis pulvinata Bohlin (Hormotilaceae, Chlorophyta): external morphology and occurrence in lotic ecosystems of the southeastern region of Brazil). Genus Ecballocystis was proposed by Bohlin in 1897 starting from the description of type species Ecballocystis pulvinata, gathered in Brazil (Rio Grande do Sul State). Genus is considered of rare occurrence in the world. In Brazil, the species E. pulvinata has been cited more frequently. Several specimens identified as E. pulvinata gathered from different streams were taxonomically evaluated through morphometrical studies, and the occurrence of the species in relation to some environmental variables was verified. Considerable morphological amplitude was verified in the populations evaluated, probably related to the environmental variations. The characteristic that varied more was the chloroplasts number, suggesting that this attribute is not a reliable taxonomic character. All samples of $E$. pulvinata were gathered in rocky bed and fast current streams $\left(38.5-75.0 \mathrm{~cm} \mathrm{~s}^{-1}\right)$ indicating that these habitat conditions are the most appropriate to its development.
\end{abstract}

Key words: Brazil, Ecballocystis, morphometry, stream, taxonomy

RESUMO - (Ecballocystis pulvinata Bohlin (Hormotilaceae, Chlorophyta): morfologia externa e ocorrência em ambientes lóticos da região Sudeste do Brasil). O gênero Ecballocystis foi proposto por Bohlin, em 1897, a partir da descrição da espécie Ecballocystis pulvinata coletada no Brasil (Rio Grande do Sul). O gênero é considerado de rara ocorrência no mundo. No Brasil, E. pulvinata tem sido citada com maior frequência. Diversos espécimes de E. pulvinata provenientes de diferentes riachos foram avaliados taxonomicamente por meio de estudos morfométricos e verificou-se a ocorrência desta espécie em relação a algumas variáveis ambientais. Considerável amplitude morfológica foi constada nas populações avaliadas, provavelmente relacionada às variações do ambiente. A característica que mais variou foi o número de cloroplastos, sugerindo que este atributo não seja um caráter taxonômico confiável. A presença de E. pulvinata em riachos de leito rochoso e correnteza rápida $\left(38,5-75,0 \mathrm{~cm} \mathrm{~s}^{-1}\right)$, mostra que, provavelmente, este tipo de hábitat seja o mais adequado ao seu desenvolvimento. Palavras-chave: Brasil, Ecballocystis, morfometria, riacho, taxonomia

\section{Introdução}

O gênero Ecballocystis foi proposto por Bohlin em 1897 a partir da descrição da espécie Ecballocystis pulvinata coletada no Brasil, no Rio Grande do Sul. As principais características desse gênero consistem na disposição oblíqua das células-filhas dentro da matriz gelatinosa; fixadas pela mucilagem às paredes das células-mãe, cujas paredes celulares remanescem na matriz gelatinosa; e a formação de colônias dendróides (Iyengar 1932, Liu \& Hu 2005, Bicudo \& Menezes 2006). Posteriormente, Yendo (1903) fez referência a algumas espécies marinhas para o gênero Ecballocystis, mas que, segundo Printz (1927), deveriam ser colocadas junto ao gênero Collinsiella Setchell \& Gardner. Espécies de Ecballocystis são essencialmente de água doce, sendo encontradas principalmente em riachos ou rios de áreas montanhosas com leitos rochosos ou próximos a quedas d'água geralmente presos ao substrato (Jose \& Patel 1990). Atualmente, este gênero pertence à família Hormotilaceae da ordem Chlorococcales (Rino \& Santos 1968, Jose \& Patel 1990, Bicudo \& Menezes 2006) e conta com 5 espécies, 5 variedades e 2 formas (Liu \& Hu 2005).

1. Universidade Estadual Paulista (UNESP), Departamento de Zoologia e Botânica, Rua Cristóvão Colombo 2265, 15054-000 São José do Rio Preto, SP, Brasil

2. Autor para correspondência: pereira.j1@terra.com.br 
Considerado de rara ocorrência, o gênero Ecballocystis tem sido citado para o Brasil (Bohlin 1897, Branco \& Necchi Junior 1996, Necchi Junior et al. 2000, Bicudo \& Menezes 2006, Krupek et al. 2008, Peres et al. 2008, Branco et al. 2009), África do Sul (Fritsch 1918), Índia (Iyengar 1932, Sankaran 1980, Jose \& Patel 1990), Portugal (Rino \& Santos 1968), França (Bourrely \& Couté 1986) e, recentemente, para a China (Liu \& Hu 2005). No Brasil, a espécie pulvinata tem sido encontrada com maior frequência, principalmente em regiões de Mata Atlântica dos Estados de São Paulo (Branco \& Necchi Junior 1996, Necchi Junior et al. 2000) e Paraná (Krupek et al. 2008, Peres et al. 2008, Branco et al. 2009). Dessa maneira, este trabalho pretende, por meio de diversos espécimes identificados como E. pulvinata, coletados em diferentes localidades, efetuar uma avaliação taxonômica por meio de estudos morfométricos e verificar a ocorrência desta espécie em relação a algumas variáveis ambientais em ambientes lóticos tropicais.

\section{Material e métodos}

Aanálise da variação morfométrica em populações de Ecballocystis pulvinata Bohlin foi realizada a partir de amostras coletadas em sete localidades diferentes. Todas as amostras avaliadas encontravam-se preservadas em solução aquosa de formaldeído $4 \%$ e são originárias de coletas feitas em: 1) Córrego da Água Limpa, município de Bady Bassitt/SP (2054'1"'S e 49²8'09'W) - Água Limpa; 2) Córrego do Pendera, município de Poloni/SP (2049'33,8"S e 4948'17'W) - Pendera; 3) Rio Preto, município de Pontes Gestal/SP (20¹1'13"'S e 49\%41'03'W) - R. Preto; 4) Córrego do Cocho, município de Cedral/ SP (2058'37' 'S e 49²0'23'W) - C. Cocho; 5) Mata Atlântica paulista (Branco \& Necchi Junior 1996) MA; 6) Floresta subtropical (Necchi Junior et al. 2000) - F. Sub Tropical e 7) Córrego Chapéu de Sol, Serra do Cipó/MG (19¹8’08'S e 43³6’04”W) - Cipó.

O talo desta alga é constituído por células agrupadas em uma matriz gelatinosa consistente. Assim, 30 células foram analisadas sob microscopia de luz quanto às seguintes características: i) diâmetro, ii) comprimento, iii) relação comprimento/largura ( $\mathrm{RC} / \mathrm{L})$, iv) número de cloroplastos e v) diâmetro de 30 colônias (diâmetro do talo). Além dessas características, a presença ou ausência de lamelação (restos de parede celular das células-mãe) foram observadas nas células analisadas. Em todas as observações e registros foi utilizado um microscópio óptico Jenaval equipado com objetiva micrometrada, câmara clara e sistema fotográfico.

Para avaliar as possíveis fontes de variação dos caracteres morfométricos e estabelecer um provável padrão de distribuição de E. pulvinata em ambientes lóticos, alguns dados referentes às características físicas e químicas dos ambientes foram coletados. As seguintes variáveis ambientais foram medidas: condutividade $\left(\mu \mathrm{S} \mathrm{cm}^{-1}\right)$, temperatura da água $\left({ }^{\circ} \mathrm{C}\right)$, turbidez (UNT) e pH utilizando-se o controlador de qualidade de água HORIBA U-10. A velocidade da correnteza $\left(\mathrm{cm} \mathrm{s}^{-1}\right)$ foi amostrada próxima ao crescimento algal empregando-se o fluxômetro mecânico General Oceanics 2030R. Informações sobre a profundidade e o tipo de substrato também foram tomadas.

Por meio dos programas estatísticos Minitab versão 10.1 e KCS (Kovach Computing Services) Multivarite Statistical Package (MVSP versão 3.1), os dados de variação morfométrica foram submetidos a análises descritivas (média e desvio padrão), análise de variância (Anova - uma via), teste de Tukey e análise de grupamento (Coeficiente de similaridade de Gower), para verificação das diferenças entre as características morfométricas nas diferentes populações e sua possível relação com a taxonomia da espécie. Os dados das variações morfométricas e morfológicas foram, ainda, submetidos à análise de correlação ( $\mathrm{r}$ de Pearson) com as características ambientais avaliadas.

\section{Resultados e Discussão}

As sete populações de Ecballocystis pulvinata Bohlin exibiram ampla variação morfométrica (tabela 1). Além disso, em todas as populações amostradas, a presença de lamelação nas células foi evidente (figura 1). A Anova mostrou que o diâmetro celular nas populações foi diferente $(\mathrm{F}=5,49 ; p<0,001)$, bem como o comprimento celular $(\mathrm{F}=9,82 ; p<0,001), \mathrm{RC} / \mathrm{L}(\mathrm{F}=4,08 ; p<0,001)$, número de cloroplastos $(\mathrm{F}=14,87 ; p<0,001)$ e $\mathrm{o}$ diâmetro do talo $(\mathrm{F}=4,92 ; p<0,001)$. Diferenças dessa natureza são relativamente comuns em macroalgas de ambientes lóticos e estão geralmente relacionadas às variações ambientais (e.g. Branco \& Necchi Junior 1998, Necchi Junior et al. 1999, Vieira Júnior \& Necchi Junior 2002, Branco 2004, Pereira \& Branco 2005).

$\mathrm{O}$ teste de Tukey demonstrou que, em relação ao comprimento celular, as células da população 


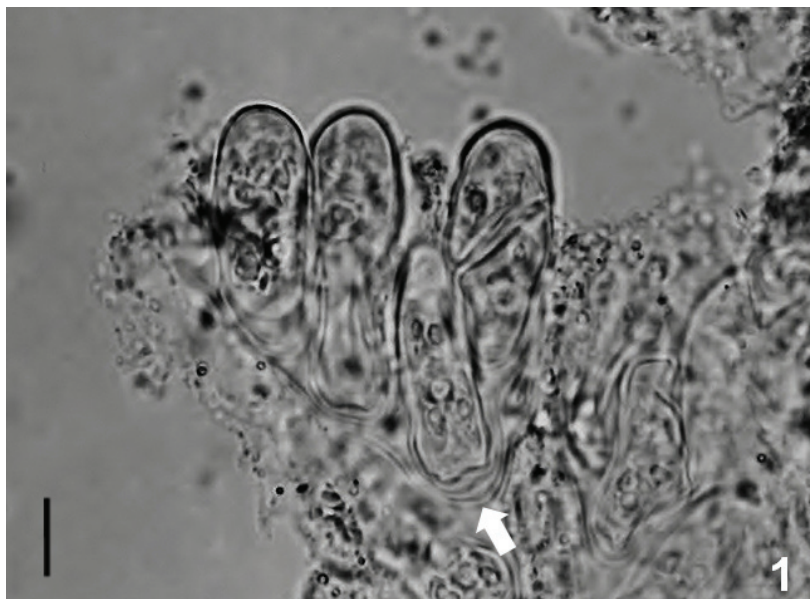

Figura 1. Ecballocystis pulvinata Bohlin. Células apresentando lamelações (seta). Barra $=10 \mu \mathrm{m}$.

Figure 1. Ecballocystis pulvinata Bohlin. Lamellation in cells (arrow). Bar $=10 \mu \mathrm{m}$.

do Córrego do Pendera foram significativamente mais longas que as células das demais populações $(\mathrm{dms}=3,75)$. Já o diâmetro celular, dessa mesma população (Pendera) diferenciou-se apenas das populações Água Limpa e Cipó, pois suas células eram estatisticamente mais largas $(\mathrm{dms}=1,43)$. Todavia, ainda em relação ao diâmetro celular, a população Água Limpa também mostrou ser diferente da população coletada na Mata Atlântica, já que as células desta última eram maiores que as de Água Limpa $(\mathrm{dms}=1,43)$. Quanto a $\mathrm{RC} / \mathrm{L}$, a única população apontada pelo teste de Tukey como diferente $(\mathrm{dms}=0,33)$ foi a aquela amostrada no Córrego Pendera, por apresentar o maior valor de $\mathrm{RC} / \mathrm{L}$, distinto das populações coletadas no córrego do Cocho e na Mata Atlântica; sendo que está última, por apresentar um valor de $\mathrm{RC} / \mathrm{L}$ alto, distinguiu-se da população Água Limpa $(\mathrm{dms}=0,33)$. O diâmetro do talo (colônias), de acordo com o teste em questão, mostrou que apenas a população coletada na Serra do Cipó diferenciou-se das provenientes do Córrego do Cocho, Mata Atlântica e do Rio Preto, por apresentar as maiores colônias $(\mathrm{dms}=0,42)$. No entanto, o número de cloroplastos foi a característica que mais variou entre as populações, pois o teste usado evidenciou diferenças significativas entre cinco das sete populações amostradas $(\mathrm{dms}=0,80)$. As características morfométricas de E. pulvinata correlacionadas com as variáveis ambientais apresentaram como significativas as seguintes correlações: diâmetro das células com turbidez $(\mathrm{r}=0,787 ; p<0,05)$ e número de cloroplastos com profundidade $(\mathrm{r}=0,767 ; p<0,05)$. Variações morfológicas e/ou morfométricas entre indivíduos da mesma espécie, mas de populações distintas são, até certo ponto, comuns entre alguns grupos algais, mas suficientes para as mesmas manterem sua identidade taxonômica, sendo que parte dessas variações parece estar relacionada com as condições ambientais (Necchi Junior \& Pascolato 1995, Vieira Júnior \& Necchi Junior 2002, Branco 2004, Pereira \& Branco 2005). Assim, algumas correlações significativas entre caracteres morfométricos e dados ambientes (diâmetro das células $\times$ turbidez e número de cloroplastos $\times$ profundidade) sugerem essa relação.

Sankaran (1980) verificou, realizando estudos em Ecballocystis fritschii Iyengar, uma ampla variação no tamanho das colônias, possivelmente influenciada pela correnteza; e no número de cloroplastos dessa espécie. Ao analisarem o microhábitat de Compsopogon coeruleus (Balbis) Montagne, Necchi Junior et al. (1999) verificaram que as variações morfométricas e suas relações com as variáveis físicas do ambiente revelaram algumas características particulares para cada população, indicando adaptações específicas que, provavelmente, contribuíram para a ampla distribuição de C. coeruleus em riachos brasileiros. Estudos envolvendo o microhábitat de populações das algas verdes Chaetophora elegans (Roth) C. Agardh e Stigeoclonium helveticum Vischer efetuados por Branco \& Necchi Junior (1998) mostraram que tanto as populações de C. elegans quanto as de S. helveticum exibiram pouca variação morfológica, mas, mesmo assim, essas variações estavam relacionadas às condições ambientais (profundidade, velocidade da correnteza e irradiância). Além disso, Pereira \& Branco (2005) sugeriram que a amplitude morfológica encontrada em Schizomeris leibleinii Kützing foi mais acentuada em populações de localidades diferentes que nas mesmas populações avaliadas ao longo do tempo, possivelmente em virtude de fatores ambientais.

A análise de grupamento das populações (figura 2) baseada na variação das características morfométricas (tabela 1) revelou a formação de quatro grupos. O grupo A compreendeu as populações do Córrego da Água Limpa (Água Limpa) e da Serra do Cipó (Cipó), com $77,5 \%$ de similaridade. Já o grupo C incluiu as populações coletadas na Floresta Subtropical (F. Sub Tropical), no Rio Preto (R. Preto) e no Córrego do Cocho (C. Cocho), com 86,9\% de semelhança entre essas populações. As populações provenientes da Mata Atlântica e do Córrego Pendera (Pendera) não 
se agruparam com quaisquer das populações citadas no nível de similaridade adotado $(75 \%)$, constituindo grupos distintos e denominados, respectivamente, B e D.

Embora a análise de grupamento tenha evidenciado grupos distintos a partir de ampla variação morfométrica, as populações apresentaram mais de $60 \%$ de similaridade das características analisadas. Estes dados concordam com as análises feitas por Pereira \& Branco (2005) para a confirmação da identidade taxonômica da espécie Schizomeris leibleinii, onde os autores sugeriram que a fonte das variações pode estar associada às condições do ambiente e ao estágio de desenvolvimento do organismo.

Tabela 1. Variáveis morfométricas (médias \pm desvio padrão; $\mathrm{n}=30$ ) observadas em populações de Ecballocystis pulvinata Bohlin. R C/L: Relação comprimento/largura.

Table 1. Morphometrical variables (mean \pm standard deviation; $\mathrm{n}=30$ ) observed in populations of Ecballocystis pulvinata Bohlin. RC/L: Relationship lenght/width.

\begin{tabular}{lccccc}
\hline \multirow{2}{*}{ Populações } & \multicolumn{5}{c}{ Características } \\
\cline { 2 - 6 } & $\begin{array}{c}\text { Diâmetro } \\
\text { Celular }(\mu \mathrm{m})\end{array}$ & $\begin{array}{c}\text { Comprimento } \\
\text { celular }(\mu \mathrm{m})\end{array}$ & $\mathrm{R} \mathrm{C} / \mathrm{L}$ & No. Cloroplastos & $\begin{array}{c}\text { Tamanho talo } \\
(\mathrm{mm})\end{array}$ \\
\hline Água Limpa & $11,3( \pm 1,56)$ & $27,8( \pm 4,86)$ & $2,5( \pm 0,49)$ & $4,2( \pm 0,76)$ & $1,1( \pm 0,71)$ \\
Cipó & $11,4( \pm 1,28)$ & $26,4( \pm 5,53)$ & $2,3( \pm 0,44)$ & $5,7( \pm 0,74)$ & $1,5( \pm 0,79)$ \\
Cocho & $12,7( \pm 2,03)$ & $27,3( \pm 3,87)$ & $2,2( \pm 0,33)$ & $4,0( \pm 0,85)$ & $0,8( \pm 0,36)$ \\
Fl. Sub Tropical & $12,4( \pm 2,30)$ & $27,7( \pm 5,56)$ & $2,3( \pm 0,61)$ & $5,1( \pm 1,67)$ & $0,9( \pm 0,49)$ \\
Mata Atlântica & $12,9( \pm 1,84)$ & $26,9( \pm 4,96)$ & $2,1( \pm 0,34)$ & $6,1( \pm 1,50)$ & $1,0( \pm 0,50)$ \\
Pendera & $13,7( \pm 2,53)$ & $34,9( \pm 5,77)$ & $2,6( \pm 0,43)$ & $4,7( \pm 0,78)$ & $1,1( \pm 0,54)$ \\
Rio Preto & $12,8( \pm 1,85)$ & $29,3( \pm 4,98)$ & $2,3( \pm 0,49)$ & $4,6( \pm 0,96)$ & $0,9( \pm 0,42)$ \\
\hline
\end{tabular}

O número de cloroplastos é usado como uma das características diferenciais entre espécies de Ecballocystis; no entanto, a partir da correlação encontrada e das verificações feitas na literatura (e.g. Iyengar 1932, Rino \& Santos 1968, Sankaran 1980, Bourrely \& Couté 1986, Jose \& Patel 1990, Liu \& Hu 2005), é aceitável que esse atributo não seja confiável, pois varia muito até mesmo entre indivíduos da mesma espécie.

As populações de E. pulvinata foram encontradas crescendo sob diversas condições ambientais (tabela 2). No entanto, todas ocorreram em hábitats cuja luz penetrava com facilidade (aberto), com substrato rochoso, profundidade média de 3 a $15 \mathrm{~cm}$ e de correnteza rápida $\left(38,5-75,0 \mathrm{~cm} \mathrm{~s}^{-1}\right)$. Dados ecológicos sobre essa espécie são escassos, contudo essas informações concordam com as investigações realizadas por Branco \& Necchi Junior (1996), Necchi Junior et al.(2000), Krupek et al. (2008), Peres et al. (2008) e Branco et al. (2009).
A maioria das espécies de Ecballocystis foi coletada crescendo em riachos apresentando leito rochoso e correnteza rápida (Iyengar 1932, Sankaran 1980, Jose \& Patel 1990, Branco \& Necchi Junior 1996, Necchi Junior et al. 2000, Liu \& Hu 2005, Krupek et al. 2008, Peres et al. 2008, Branco et al. 2009). Dessa maneira, é possível inferir que esse tipo de hábitat seja o mais adequado ao desenvolvimento de E. pulvinata. De acordo com Burkholder (1996), o leito rochoso de riachos é um substrato estável, permitindo sua intensa colonização algal. E, de um modo geral, algas com o talo gelatinoso parecem ser favorecidas pela velocidade da correnteza, pois este fator permite maior absorção de nutrientes devido à redução no tamanho da concha de difusão (Stevenson 1996, Branco \& Necchi Junior, 2003).

Ecballocystis pulvinata não apresenta ampla distribuição na natureza, sendo considerada rara. Talvez, isso ocorra devido ao tamanho diminuto de suas colônias gelatinosas, permitindo que essa alga 
seja negligenciada em determinados estudos (a menos que a sua biomassa seja elevada), ou em virtude do dessecamento de riachos já que a mesma é encontrada em áreas pouco profundas $(3-15 \mathrm{~cm})$. Ou, ainda, mediante as condições estreitas de microhábitat como verificado em Chaetophora elegans por Branco \&
Necchi Junior (1998), já que E. pulvinata apresenta um hábitat semelhante ao de C. elegans. Acredita-se, também, que a escassez de estudos envolvendo os ambientes lóticos, bem como a carência de taxonomistas, possam ter contribuído com os poucos registros dessa alga nesses ambientes.

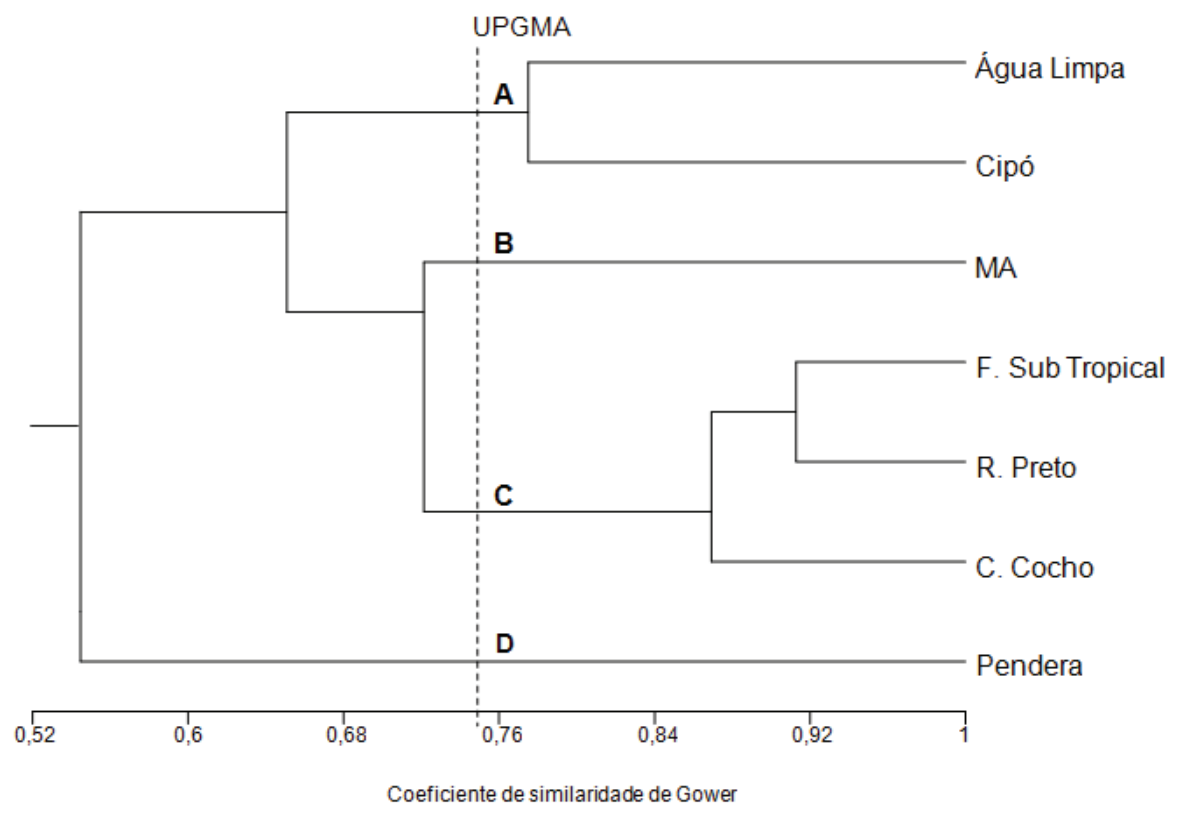

Figura 2. Análise de grupamento das características morfométricas em Ecballocystis pulvinata Bohlin de diferentes populações (Coeficiente de similaridade de Gower).

Figure 2. Cluster analysis based on morphometrical variables from different populations of Ecballocystis pulvinata Bohlin (Gower similarity coefficient).

Tabela 2. Características ambientais selecionadas para as populações de Ecballocystis pulvinata Bohlin. (valores médios) Table 2. Selected environmental characteristics of Ecballocystis pulvinata populations Bohlin. (means values)

\begin{tabular}{lcccccc}
\hline \multirow{2}{*}{ População } & \multicolumn{7}{c}{ Variáveis ambientais } \\
\cline { 2 - 7 } & $\begin{array}{c}\text { Condutividade } \\
\left(\mu \mathrm{S} \mathrm{cm}^{-1}\right)\end{array}$ & $\begin{array}{c}\text { Temperatura } \\
\left({ }^{\circ} \mathrm{C}\right)\end{array}$ & $\mathrm{pH}$ & Turbidez $(\mathrm{UNT})$ & $\begin{array}{c}\text { Velocidade } \\
\left(\mathrm{cm} \mathrm{s}^{-1}\right)\end{array}$ & $\begin{array}{c}\text { Profundidade } \\
(\mathrm{cm})\end{array}$ \\
\hline Água Limpa & 116,0 & 24,0 & 7,0 & 22,0 & 52,0 & 4,0 \\
Cipó & 11,2 & 20,9 & 6,3 & 1,0 & 38,5 & 9,8 \\
Cocho & 30,0 & 27,2 & 7,2 & 13,0 & 42,3 & 6,0 \\
F. Sub Tropical & 12,0 & 17,4 & 6,9 & 5,0 & 50,0 & 15,0 \\
Mata Atlântica & 30,6 & 19,8 & 7,2 & 3,6 & 46,0 & 15,0 \\
Pendera & 40,0 & 26,0 & 7,1 & 34,0 & 40,0 & 5,0 \\
Rio Preto & 90,8 & 17,1 & 6,8 & 8,7 & 75,0 & 3,0 \\
\hline
\end{tabular}




\section{Literatura citada}

Bicudo, C.E.M. \& Menezes, M. 2006. Gêneros de algas de águas continentais do Brasil: chave para identificação e descrições. 2 ed. Rima, São Carlos.

Bohlin, K. 1897. Die Algen der Ersten Regnell'schen Expedition. I. Protococcoideen. Bihang til Kongliga Svenska Vetenskaps Akademiens Handlingar 23: 3-47.

Bourrely, P. \& Couté, A. 1986. Algues d'eau douce de l'ile de La Réunion (Diatomées exclue). Cryptogamie, Algologie 7: 87-121.

Branco, L.H.Z. 2004. Morphometrical variations in two cyanoprokaryote populations from tropical streams. Archiv für Hydrobiologie Supplementaband Algological Studies 113: 101-116.

Branco, C.C.Z. \& Necchi Junior, O. 2003. Temporal dynamics of two species of Chaetophoraceae (Chlorophyta) in tropical streamsn of São Paulo State, southeastern Brazil. Revista Brasileira de Botânica 23: 151-161.

Branco, C.C.Z. \& Necchi Junior, O. 1998. Microhabitat and morphometric variation of two Chaetophoracean (Chaetophorales, Chlorophyta) species in tropical streams of southeastern Brazil. Phycological Research 46: 169-174.

Branco, C.C.Z. \& Necchi Junior, O. 1996. Survey of stream macroalgae of eastern Atlantic Rainforest of São Paulo State, southeastern Brazil. Archiv für Hydrobiologie Supplementaband Algological Studies 80: 35-57.

Branco, C.C.Z., Peres, C.K., Krupek, R.A. \& Bertusso, F.R. 2009. Macroalgas de ambientes lóticos da região Centro-Oeste do Paraná, região Sul do Brasil. Biota Neotropica 9: 227-235.

Burkholder, J.M. 1996. Interactions of benthic algae with their substrata. In: R.J. Stevenson, M.L. Bothwell \& R.L. Lowe (eds.). Algal ecology: freshwater benthic ecosystems. Academic Press, San Diego, pp. 253-289.

Fritsch, F.E. 1918. Contributions to our knowledge of freshwater algae, mostly from the Cape peninsula. In the Herbarium of South African Museum. Annals South African Museum 9: 483-611.

Iyengar, M.O.P. 1932. Two little known genera of green algae (Tetrasporidium and Ecballocystis). Annals of Botany 46: 191-227.

Jose, L. \& Patel, R.J. 1990. Ecballocystis ramosa f. minor Bourrely et Couté, a rare green alga from India. Cryptogamie, Algologie 11: 305-308.
Krupek, R.A., Branco, C.C.Z. \& Peres, C.K. 2008. Levantamento florístico das comunidades de macroalgas da bacia do Rio das Pedras, região centro-sul do Estado do Paraná, Sul do Brasil. Hoehnea 35: 189-208.

Liu, G.-X. \& Hu, Z.-Y. 2005. Ecballocystis hubeiensis sp. nova (Chlorococcales, Chlorophyceae) from China. Archiv für Hydrobiologie Supplementaband Algological Studies 116: 39-47.

Necchi Junior, O. \& Pascoaloto, D. 1995. Morphometry of Compsopogon coeruleus (Compsopogonaceae, Rhodophyta) populations in a tropical river basin of southeastern Brazil. Archiv für Hydrobiologie Supplementaband Algological Studies 76: 61-73.

Necchi Junior, O., Branco, C.C.Z. \& Gomes, R.R.V. 1999. Microhabitat and plant structure of Compsopogon coeruleus (Compsopogonaceae, Rhodophyta) populations in streams from São Paulo State, southeastern Brazil. Cryptogamie, Algologie 20: 75-87.

Necchi Junior, O., Branco, C.C.Z. \& Branco, L.H.Z. 2000. Distribution of stream macroalgae in São Paulo, southeastern Brazil. Archiv für Hydrobiologie Supplementaband Algological Studies 97: 91-112.

Pereira, J.L. \& Branco, L.H.Z. 2005. Aspectos taxonômicos em Schizomeris leibleinii Kützing (Chaetophorales, Chlorophyta). Revista Brasileira de Botânica 28: 305-318.

Peres, C.K., Branco, C.C.Z. \& Krupek, R.A. 2008. Macroalgas de riachos da Serra da Prata, leste do Paraná, Sul do Brasil. Acta Botanica Brasilica 22: 333-344.

Printz, H. 1927. Chlorophyceae. In: A. Engler \& K. Prantl (eds.). Die näturlichen Pflanzenfamilien Wilhelm Engelmann, Leipzig, v.3, pp.78-79.

Rino, J.A. \& Santos, M.F. 1968. Subsídios para o conhecimento das algas de Portugal - III. Anuário da Sociedade Broteriana 34: 19-56.

Sankaran, V. 1980. A rare green alga Ecballocystis fritschii from Anamalays. Phykos 19: 1-7.

Stevenson, R.J. 1996. The stimulation and drag force of current. In: R.J. Stevenson, M.L. Bothwell \& R.L. Lowe (eds.). Algal ecology: freshwater benthic ecosystems. Academic Press, San Diego, pp. 321-340.

Vieira Júnior, J. \& Necchi Junior, O. 2002. Microhabitat and plant structure of Characeae (Chlorophyta) populations in streams from São Paulo State, southeastern Brazil. Cryptogamie, Algologie 23: 51-63.

Yendo, K. 1903. Three marine species of Ecballocystis. Botanical Magazine (Tokyo) 17: 199-206. 